\title{
Mantle Cell Lymphoma Case Report
}

\author{
V.I. Podzolkov T.S. Vargina A.E. Pokrovskaya T.A. Safronova \\ A.A. Abramova
}

Federal State Autonomous Educational Institution of Higher Education, I.M. Sechenov

First Moscow State Medical University of the Ministry of Health of the Russian Federation

(Sechenov University), Moscow, Russia

\section{Keywords}

Lymphoproliferative diseases · Non-Hodgkin's lymphomas · Mantle cell lymphoma ·

Immunophenotyping of the tumor · Cyclin D1

\begin{abstract}
Introduction: Due to the beginning of the use of immunophenotypic and cytogenetic techniques, new nosological forms of lymphoproliferative diseases have appeared over the past few decades. According to the WHO classification (2008), today there are more than 50 known lymphoproliferative diseases. Case Presentation: We present the case of a 51-year-old man with lymphoproliferative syndrome. Our patient underwent morphological and immunohistochemical investigations of biopsy materials from the right inguinal lymph node. The morphological picture was characteristic for small cell lymphoma. Immunophenotypically, tumor proliferate cells expressed CD20, CD76b, CD5, and cyclin D, and the tumor immunophenotype matched mantle cell lymphoma. Discussion: At the present stage of the development of medicine, the diagnosis of lymphoproliferative diseases is based on the clinical picture of the disease with the definition of localization and characteristics of the tumor process, morphological study of tumor tissue and cells, and immunophenotypic and/or cytogenetic analyses are mandatory to determine the final diagnosis.

(C) 2018 The Author(s)

Published by S. Karger AG, Basel




\section{Case Reports in Oncology}

\section{Introduction}

Historically, lymphomas were classified into two groups: Hodgkin's lymphoma and nonHodgkin's lymphomas. Hodgkin's lymphoma (previously - disease) was described almost 200 years ago (T. Hodgkin, 1832; the term was suggested by S. Wilks in 1865) and became the first nosologically defined neoplastic disease of lymphoid tissue and the only one which has been preserved in an almost unchanged form to the present day. In the professional sphere, the term "non-Hodgkin's lymphomas" was adopted. It is lacking in the classification but is widely used to denote all lymphomas with the exception of Hodgkin's lymphoma [1].

Since the end of the last century, the introduction of immunophenotypic and cytogenetic techniques has led to an improvement in diagnosis and the development of new nosologies of lymphoproliferative diseases. Currently, the WHO classification (2008) is the only generally accepted classification of tumors of hematopoietic and lymphoid tissue [2]. The WHO classification (2008) on lymphoproliferative diseases is divided into 5 main sections: tumors originating from the lymphoid cell precursors, mature B-cell tumors, mature T- and NK-cell tumors, Hodgkin's lymphoma, and post-transplant lymphoproliferative diseases.

In fact, the WHO classification is a list of more than 50 nosological forms of lymphoproliferative diseases, formulated on the basis of a consensus of pathologists and hematologists/oncologists. Nosologically independent forms of diseases are those with a characteristic stable set of morphological, immunological (immunophenotypic), genetic and clinical signs [1].

A clinical observation is provided as a demonstration of diagnosis of lymphoproliferative disease.

\section{Case Presentation}

Patient G., 51 years old, was admitted with complaints about shortness of breath, dizziness, and episodes of palpitations after minimal physical exertion, sweating, subfebrile body temperature up to $37.5^{\circ} \mathrm{C}$, general weakness, and increased fatigue.

\section{Anamnesis Vitae}

Bad habits: has smoked 1 pack of cigarettes per day for more than 30 years (smoker's index is 30 pack years). Occupational hazards: negative; he works as an auto mechanic. Comorbidities: duodenal ulcer during the last 8 years, for which he receives periodical treatment with Ranitidine.

\section{Anamnesis Morbi}

After the acute respiratory viral infection, the patient noted the appearance of weakness, dyspnea, episodes of dizziness, and rapid heart rate. Two weeks later, according to the patient, "bumps on the body" (generalized lymphadenopathy) and "bruises" (hemorrhages) and nosebleeds appeared. The patient lost weight (he could not specify the number of kilos). He was admitted to a polyclinic where he was diagnosed with severe anemia (hemoglobin $60 \mathrm{~g} / \mathrm{L}$ ). He was referred for hospitalization. 


\section{Case Reports in Oncology}

\section{Status Praesens}

The general condition of the patient is of moderate severity. Generalized lymphadenopathy was noted (Fig. 1): enlargement of submaxillary, dorsal cervical, axillary, inguinal lymph nodes on both sides, with dense elastic consistency, painless, not soldered to surrounding tissues, the skin over them is not changed.

On the limbs, small hemorrhagic eruptions were noted. In the lungs, vesicular breathing with harsh accent without rales. Breathe rate -20 per min. The borders of the heart are within normal limits. Auscultation of the heart: heart sounds are moderately muffled, the rhythm is regular, tender systolic murmurs at all points of auscultation were heard. Heart rate $-96 \mathrm{bpm}$. Blood pressure: $100 / 70 \mathrm{~mm} \mathrm{Hg}$ on both arms. The liver is $2-3 \mathrm{~cm}$ below the edge of the right rib arch, spleen $-2 \mathrm{~cm}$ below the edge of the left rib arch.

Table 1 shows the laboratory changes detected during the examination. Complete blood count was performed on the first day of hospitalization and was repeated on the sixth day of hospitalization.

In general, blood analysis was performed with an interval of 5 days; severe normochromic anemia and significant leukocytosis were observed. In the leukocyte formula, proliferation of lymphocyte lineage was noted due to an increase in the number of lymphocytes and prolymphocytes. Stab neutrophils were not detected, segmented neutrophil level was significantly reduced, blast cells were found. In addition, expressed thrombocytopenia and increased ESR were found. RW, HIV, HbsAg, anti-HCV tests were negative.

Due to anemia, serum iron, vitamin $B_{12}$, and folic acid tests were performed. All parameters were within the normal range. Severe anemia was regarded as anemia due to the underlying disease.

Biochemical blood test, urinalysis, ECG, chest X-ray, and EHDS results did not show pathological changes.

Abdominal cavity and kidneys ultrasound: lymphadenopathy (enlarged lymph nodes in the area of hepatic and splenic hilum, in the small omentum, mesenteric, along the abdominal aorta and inferior vena cava, and iliac lymph nodes). Hepatosplenomegaly. Diffuse changes in the liver and pancreas.

Ultrasonography of the mediastinum and pleural cavities: presence of lymph nodes in the anterior mediastinum cannot be ruled out, due to unsatisfactory visualization.

Peripheral lymph nodes ultrasound: contours are clear, even, with low echogenicity. Lymph nodes of all groups have a uniform structure, equal on both sides. Enlarged parotid, posterior cervical, submandibular, supraclavicular, subclavian, axillary, inguinal lymph nodes.

Considering the clinical picture of the disease and investigations results, a decision was made to perform bone marrow analysis (Table 2). Conclusion: leukopoiesis, shift in the direction of lymphocyte lineage cells, a large number of leukolytic cells. There are lymphocytes with split nuclei. Erythropoiesis: individual erythroblasts. Thrombocytopoiesis: megakaryocytes were not found. According to the myelogram, there is a marked proliferation of lymphoid cells with different degrees of maturity.

Considering the proliferation of lymphoid cells in the peripheral blood and bone marrow, a lymphoproliferative disease (chronic lymphocytic leukemia, follicular lymphoma with leukemia) was suspected. To establish a definitive diagnosis, a decision was made to conduct an immunohistochemical study. 
Right inguinal lymph node biopsy was performed with obtaining a sample as a substrate for immunohistochemical examination. In the finished preparations, fragments of the tissue of the lymph node with diffusely nodular proliferation of small lymphoid cells with irregular, centrocytic-like nuclei and uniformly dispersed chromatin structure were observed. Perivascular sclerosis, lymphoid infiltration of perinodal tissue were noted. Conclusion: morphological picture of small cell lymphoma.

In order to verify the type of lymphoma on paraffin block sections, an immunohistochemical study of the lymph node was performed using antibodies to CD3, CD5, CD10, CD20, CD23, CD79b, BCL-6, cyclin D1 (SP-4), and Ki-67. Tumor proliferate cells express CD20 (intensive membrane reaction), CD76b, CD5 (weak membrane reaction, intense in scattered T cells when compared to $\mathrm{CD} 3+$ ), and cyclin D1 (nuclear expression). The average proliferative index of Ki67 is $15-20 \%$. T-cells (CD3+) are scattered among the B-cell proliferates. In the reaction with CD23, clusters of FDC CD23+ were detected. Expression of lymphoid cells CD10, BCL-6 was not detected. Conclusion: in the lymph node, the morphological picture and immunophenotype correspond to mantle cell lymphoma (MCL), morphologically - classical variant.

\section{Clinical Diagnosis}

Primary: IVA stage MCL with the predominant impairment of all groups of peripheral, abdominal, retroperitoneal lymph nodes, bone marrow, and spleen. Severe anemia.

Concomitant disease: duodenal ulcer, remission. Chronic bronchitis associated with smoking, remission.

The treatment was performed according to R-CHOP protocol (rituximab [MabThera] 700 $\mathrm{mg} /$ i.v. drip, cyclophosphamide [Endoxan] 1,400 mg/i.v. drip on the 1st day, doxorubicin 90 $\mathrm{mg} / \mathrm{i} . \mathrm{v}$. on the 1st day, oncovir [Vincristine] $2 \mathrm{mg} / \mathrm{i}$.v. bolus on the 1st day, prednisolone 120 $\mathrm{mg} / \mathrm{i}$.v. drip on days 1-5). In addition, the patient received therapy with allopurinol $600 \mathrm{mg}$ per day, omeprazole $20 \mathrm{mg}$ b.i.d.

In the background of the therapy, positive dynamics were noted: the number of red blood cells $\left(2.9 \times 10^{12} / \mathrm{L}\right)$ and platelets $\left(60 \times 10^{9} / \mathrm{L}\right)$ increased, the number of white blood cells decreased $\left(3.3 \times 10^{9} / \mathrm{L}\right)$. The patient was discharged under the supervision of a hematologist at the place of residence in a relatively satisfactory condition. The next course of polychemotherapy was scheduled within a month.

\section{Discussion}

The term "non-Hodgkin's lymphomas" defines a heterogeneous group of malignant tumors of lymphatic tissue with specific biological, morphological and clinical features, as well as with various responses to therapy, prognosis and survival of patients. In general, today about half of such clinical and morphological types of lymphoma are potentially curable diseases. Most non-Hodgkin's lymphomas originate from tumorous analogues of B-lymphocytes, the remainder account for T-cell and a very small part for NK-cell tumors [1].

MCL is a B-cell tumor consisting of monomorphic small or medium lymphoid cells, with uneven contour of the nucleus and translocation of the CCND1 gene [1]. The most common "classical" variant of mantle cell lymphoma was first described in 1973 by the German pathologist K. Lennert [3, 4]. 
The incidence of MCL among all non-Hodgkin's lymphomas in adults is 6\%. The incidence of MCL in the United States and Europe is 2-3 per 100,000 annually. The median age of patients is 60-65 years. Men are 2-6 times more likely to be diagnosed with the disease than women. The median survival is about $3-5$ years $[1,5]$.

Leukemization of lymphoma represents the diffuse impairment of the hematopoietic organs, which arises as the pathological process progresses. Lymphomas with leukemization are follicular lymphoma, marginal zone lymphoma, and MCL $[1,6]$. The incidence of bone marrow damage and leukemization of MCL is $20-80 \%$ [1].

The most important pathogenetic stage of MCL development is translocation of genes encoding cyclin D1 (CCND1) and heavy chains of immunoglobulins - $t(11 ; 14)(q 13 ; q 32)$. Therefore, the main diagnostic criteria of MCL are the detection of cyclin D1 in immunohistochemical studies or the detection of $\mathrm{t}(11 ; 14)(\mathrm{q} 13 ; \mathrm{q} 32)$ by the D-FISH method $[1,4]$. In patient G., immunohistochemical study of the right inguinal lymph node material was performed, where tumor proliferate cells expressed CD20, CD76b, CD5, and cyclin D1.

D group cyclins are the main regulators of cell transition from the $\mathrm{G}_{1}$ phase of the cell cycle to the $\mathrm{S}$ phase. In normal lymphocytes, this function is performed by cyclin D3, and in cells of MCL - cyclin D1. Pathologically increased amount of cyclin D1 allows omitting the apoptosis control point by the cytogenetically defective cells in the $\mathrm{G}_{1}$ phase of the cell cycle, which gives them an advantage in growth over normal cellular analogues and leads to their accumulation.

In the vast majority of cases, the tumor is characterized by an aggressive course and at the time of diagnosis, disseminated lesions of lymphatic and extranodal organs are determined, which corresponds to stage IV according to the Ann Arbor classification, which was observed in our patient. More than 70\% of patients are diagnosed with MCL in the terminal stage IV of the disease. Stage A revealed in our patient indicates the absence of systemic (constitutional) symptoms: fever of more than $38^{\circ} \mathrm{C}$, drenching, night sweating, and loss of $10 \%$ or more of the baseline body mass during 6 months [1].

Despite the sensitivity of MCL to first-line chemotherapy, subsequent relapses and multidrug resistance to cytostatic effects determine a poor prognosis. If complete remission is not obtained after induction of therapy, the patients' survival does not exceed 1.5 years [4]. Consolidation of the first remission with allogeneic bone marrow or allogeneic hematopoietic stem cell transplantation may be the only potential cure for MCL, but it is limited due to the toxicity of the procedure and the age of the patient [1]. Without a hematopoietic stem cell transplantation, the median overall survival is 3 years [4].

Until the 1970s, the diagnosis was based solely on the results of morphological (histological and cytological) investigations. When studying the reproducibility of the WHO classification, it was shown that the conclusion of hematology experts on the diagnosis coincides in 4193\% of cases when only histological criteria were used. After "blind" reevaluation, pathologists could reproduce their own diagnosis in about $85 \%$ of cases. Therefore, at the present stage, morphological research and determination of tumor immunophenotype are mandatory diagnostic procedures in all lymphoproliferative diseases [1].

The accumulated material on the use of immunophenotypic and cytogenetic techniques made it possible to more clearly define a number of lymphoproliferative diseases, including those whose nosological independence was not considered proven. Morphological examination remains the key element of diagnostics, as it allows to reveal the cellular substrate of a tumor and to determine its tissue architecture. The list of CD antigens introduced into the 
nomenclature of differentiating antigens of human leukocytes and determined by immunophenotyping is constantly updated, and currently contains about $400 \mathrm{CD}$ antigens and their subtypes [7]. Therefore, a number of new antibodies is introduced into the immunohistochemical diagnostic panels, which allow to determine the tumor immunophenotypes and increase the specificity of the morphological study.

In general, for diagnostics and classification of lymphoproliferative diseases, all available information is used, including information about the structure of tumor tissue and cells, immunophenotype, genetic characteristics of tumor cells, and localization and features of the tumor process. So, in our clinical observation, the final diagnosis of the patient G. was carried out on the basis of the clinical picture of the disease, lymphoproliferative changes in the blood and bone marrow, morphological and immunohistochemical lymph node examination with the determination of the immunophenotype of the tumor.

\section{Statement of Ethics}

The patient has given his written permission to publish his personal data (including personal images).

\section{Disclosure Statement}

The authors state that there is no conflict of interest.

\section{References}

1 Rukovitsyn OA, editor. Hematology: national guideline. Moscow: GEOTAR-Media; 2017. p. 784.

2 Swerdlow SH, Campo E, Harris NL, Jaffe ES, Pileri SA, Stein H, Thiele J, Vardiman JW, editors. WHO classification of tumours of haematopoietic and lymphoid tissues. Geneva: WHO press; 2008. p. 229-37.

3 Lennert K, Stein H, Kaiserling E. Cytologicaland functional criteria for the classification of malignant lymphoma. Br J Cancer. 1975;31:29-43.

4 Vorobyov AI, editor. Rational drug therapy of blood system diseases: a guide for practicing physicians. Moscow: GEOTAR-Media; 2009. p. 688.

5 Kluin-Nelemans H, Hoster E, Hermini O, et al. Treatment of older patients with mantle cell lymphoma. N Engl J Med. 2012 Nov 1;367(18):1765.

6 Dreyling M, Thieblemont C, Gallamini A, et al. ESMO consensus conferences: guidelines on malignant lymphoma. Part 2: marginal zone lymphoma, mantle cell lymphoma, peripheral T-cell lymphoma. Ann Oncol. 2013 Apr;24(4):857-77.

7 Available from: https://www.hcdm.org. 


\section{Case Reports in Oncology}

Case Rep Oncol 2018;11:814-821

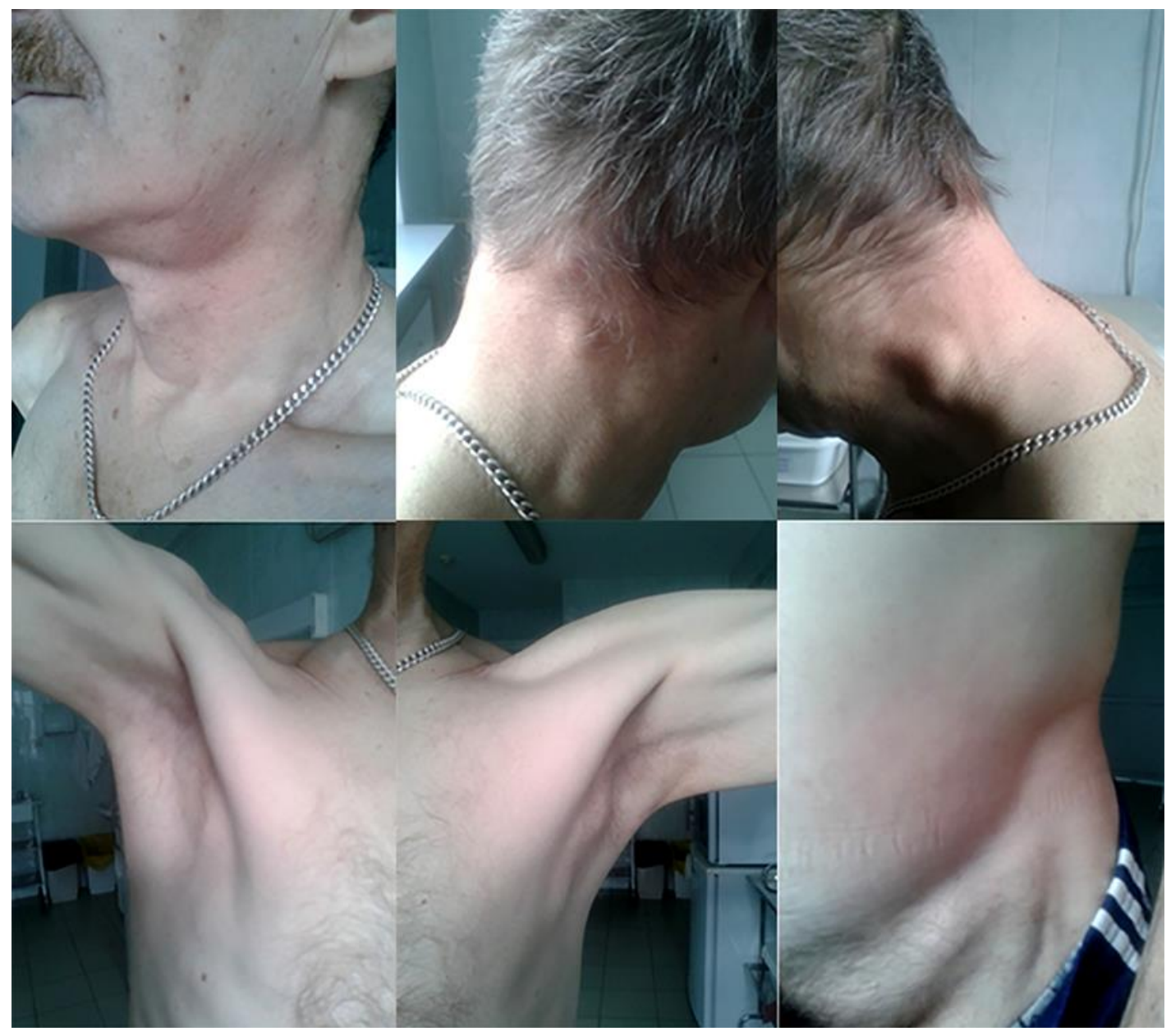

Fig. 1. Generalized lymphadenopathy. 
Table 1. Complete blood count results on the first and sixth day of hospitalization

Parameters

Hemoglobin, g/L

Red blood cells, 1012/L

Color index

Stab neutrophils, \%

Segmented neutrophils, \% 2

Platelets, $10^{9} / \mathrm{L}$

Blast cells, \%

Prolymphocytes, \%

Lymphocytes, \%

Monocytes, \%

Cytolysis cells

ESR, $\mathrm{mm} / \mathrm{h}$ 1st day of hospitalization 6th day of hospitalization

$\begin{array}{rc}47 & 41 \\ 1.58 & 1.38 \\ 0.9 & 0.9 \\ 53.9 & 46.3 \\ -\quad & - \\ 2 & 3 \\ 28 & 36 \\ 5 & 6 \\ 24 & 20 \\ 68 & 70 \\ 1 & 1 \\ -\quad & 0-1-3 \\ 52 & 48\end{array}$

(c) 2018 The Author(s). Published by S. Karger AG, Basel
www.karger.com/cro

Podzolkov et al.: Mantle Cell Lymphoma Case Report

Table 2. Myelogram

\begin{tabular}{lcc}
\hline Myelogram & Result, \% & Reference range, \% \\
\hline Blast cells & 5.0 & $1.4-1.6$ \\
Promyelocytes & - & $2-3$ \\
Neutrophilic myelocytes & - & $3.8-8.0$ \\
Neutrophilic metamyelocytes & - & $6.0-8.0$ \\
Stab neutrophils & 1.0 & $25.0-30.0$ \\
Segmented neutrophils & 6.0 & $20.0-27.0$ \\
Eosinophilic myelocytes & - & $0.1-1.0$ \\
Eosinophils & 1.0 & $1.8-3.8$ \\
Basophils & - & $0.1-1.2$ \\
Lymphocytes & 70.0 & $9.0-10.0$ \\
Prolymphocytes & 15.0 & $0-0.2$ \\
Monocytes & 2.0 & $1.0-2.0$ \\
Reticular cells & - & $2.0-3.0$ \\
Reticular plasma cells & - & $0.4-0.6$ \\
Macrophages & - & \\
Mitoses & - & \\
\hline
\end{tabular}

